\title{
On the triple points of singular maps
}

\author{
Tobias Ekholm and András Szücs
}

\begin{abstract}
The number of triple points $(\bmod 2)$ of a self-transverse immersion of a closed $2 n$ manifold $M$ into $3 n$-space are known to equal one of the Stiefel-Whitney numbers of $M$. This result is generalized to the case of generic (i.e. stable) maps with singularities. Besides triple points and Stiefel-Whitney numbers, a certain linking number of the manifold of singular values with the rest of the image is involved in the generalized equation which corrects an erroneous formula in [9].

If $n$ is even and the closed manifold is oriented then the equations mentioned above make sense over the integers. Together, the integer- and $\bmod 2$ generalized equations imply that a certain Stiefel-Whitney number of closed oriented $4 k$-manifolds vanishes. This Stiefel-Whitney number is in fact the first in a family which vanish on such manifolds.
\end{abstract}

Mathematics Subject Classification (2000). 57R20, 57R45, 58K30.

Keywords. Stable map, linking number, triple point, Stiefel-Whitney number, orientable $4 k$ manifold.

\section{Introduction}

In his classical paper [10] of 1946, Whitney showed that the number of double points of a self-transverse immersion of an $n$-manifold into $2 n$-space is related to the Euler number of its normal bundle. Since then many results of a similar nature have been found. This paper deals with a generalization of one of these results, the Herbert-Ronga formula [5] which expresses the number of triple points of a self-transverse immersion of a closed $2 n$-manifold into $3 n$-space in terms of one of its characteristic numbers. More precisely, the Herbert-Ronga formula is extended to singular generic (i.e. stable) maps of $2 n$-manifolds into $3 n$-space. (In this paper all manifolds and maps are assumed to be $C^{\infty}$-smooth, unless otherwise explicitly stated.) To state the formula, some notation is needed:

Let $M$ be a closed $2 n$-manifold and let $f: M \rightarrow \mathbb{R}^{3 n}$ be a generic map. If $\Delta(f) \subset \mathbb{R}^{3 n}$ denotes the set of double points of $f$ then $\Delta(f)$ is an immersed $n$ dimensional submanifold with boundary. The self-intersection points of $\Delta(f)$ are the triple points of $f$. The boundary of $\Delta(f)$ is $\Sigma(f)$, the set of singular values of $f$. 
Define $t_{2}(f) \in \mathbb{Z}_{2}$ as the mod 2-number of triple points of $f$. Let $\Sigma^{\prime}(f)$ denote the $(n-1)$-dimensional submanifold of $\mathbb{R}^{3 n}$ which is obtained by shifting $\Sigma(f)$ slightly along its outward normal vector field in $\Delta(f)$. Then $\Sigma^{\prime}(f) \cap f(M)=\emptyset$. Define $l_{2}(f) \in \mathbb{Z}_{2}$ as the $\bmod 2$-linking number of the cycles $f(M)$ and $\Sigma^{\prime}(f)$ in $\mathbb{R}^{3 n}$. If $i_{1}+\cdots+i_{m}=2 n$ then let $\bar{w}_{i_{1}} \ldots \bar{w}_{i_{m}}[M] \in \mathbb{Z}_{2}$ denote the product of the normal Stiefel-Whitney classes of $M$ in dimensions $i_{1}, \ldots, i_{m}$ evaluated on the fundamental homology class of $M$.

Theorem 1. Let $M$ be a closed manifold of dimension $2 n$ and let $f: M \rightarrow \mathbb{R}^{3 n}$ be a generic map. Then

$$
t_{2}(f)+l_{2}(f)=\bar{w}_{n}^{2}[M]+\bar{w}_{n+1} \bar{w}_{n-1}[M]
$$

Theorem 1 is proved in Section 2. It corrects the erroneous theorem on the second page of [9], in which the second term in the right hand side of Equation (1) is missing.

For closed oriented $4 k$-manifolds Equation (1) can be lifted to an integer equation: If $n=2 k$ is even and $M$ is oriented then there is an induced orientation on $\Delta(f)$ as well as on the triple points of $f$. Define $t(f) \in \mathbb{Z}$ as the algebraic number of triple points of $f$. The orientation of $\Delta(f)$ induces an orientation of its boundary $\Sigma(f)$ which in turn induces an orientation of $\Sigma^{\prime}(f)$. Define $l(f) \in \mathbb{Z}$ as the linking number of the oriented cycles $f(M)$ and $\Sigma^{\prime}(f)$ in $\mathbb{R}^{6 k}$. Let $\bar{p}_{k}\left[M^{4 k}\right]$ denote the $k^{\text {th }}$ normal Pontryagin number of $M$. The following theorem is Lemma 4 in $[1]$.

Theorem 2. Let $M$ be a closed oriented manifold of dimension $4 k$ and let $f: M \rightarrow$ $\mathbb{R}^{6 k}$ be a generic map. Then

$$
3 t(f)-3 l(f)=\bar{p}_{k}[M] .
$$

Equation (2) turned out to be very useful: It is used in the derivation of a geometric formula for Smale invariants of immersions of spheres, see [1] and [2], and in the study of geometric features of the regular homotopy classification of immersions of 3-manifolds in 5-space, see [7].

If $M$ is a closed oriented $4 k$-manifold then the mod 2-reduction of $\bar{p}_{k}[M]$ equals $\bar{w}_{2 k}^{2}[M]$. Hence Theorems 1 and 2 together imply that

$$
\bar{w}_{2 k+1} \bar{w}_{2 k-1}[M]=0
$$

for any closed oriented $4 k$-manifold $M$. In fact, $\bar{w}_{2 k+1} \bar{w}_{2 k-1}[M]$ is the first in a sequence of Stiefel-Whitney numbers which vanish on closed oriented $4 k$-manifolds. More precisely,

Theorem 3. (Stong). If $M$ is an oriented $4 k$-manifold and $\left(2 k_{1}+1\right)+\cdots+$ $\left(2 k_{r}+1\right)=4 k$ then

$$
\bar{w}_{2 k_{1}+1} \ldots \bar{w}_{2 k_{r}+1}[M]=0 .
$$


This theorem was communicated by R. Stong to the second author together with a proof of the first case (3). A proof of Theorem 3 is presented in Section 3.

\section{Proof of Theorem 1}

Fix a generic map $f: M \rightarrow \mathbb{R}^{3 n}$ of a closed $2 n$-manifold. Let $\tilde{\Sigma} \subset M$ denote the $(n-1)$-dimensional submanifold of singular points of $f$ and let $\Sigma=f(\tilde{\Sigma})$. Then $f$ maps $\tilde{\Sigma}$ diffeomorphically to $\Sigma$.

Let $\tilde{\Delta} \subset M$ denote the closure of the preimages of multiple points of $f$. Then $\tilde{\Delta}$ is an immersed closed $n$-dimensional manifold with transverse double points at the preimages of triple points of $f$. Let $\tilde{\Delta}_{\text {res }}$ denote the resolution of $\tilde{\Delta}$ and let $\tilde{\iota}: \tilde{\Delta}_{\text {res }} \rightarrow M$ denote the natural immersion with image $\tilde{\Delta} \subset M$.

There is a natural involution $T: \tilde{\Delta}_{\text {res }} \rightarrow \tilde{\Delta}_{\text {res }}$ such that $f \circ \tilde{\iota} \circ T=f \circ \tilde{\iota}$. Since no triple point of $f$ is singular we have a natural embedding $\tilde{\Sigma} \subset \tilde{\Delta}_{\text {res }}$ and $\tilde{\Sigma}$ is the fix point set of $T$.

Let $\nu(\tilde{\iota})$ denote the normal bundle of the immersion $\tilde{\iota}$ and let $\nu$ denote its restriction to $\tilde{\Sigma}$. Since $\nu$ is an $n$-dimensional vector bundle over an $(n-1)$-manifold there exists a non-zero section. Let $\tilde{s}$ be such a section.

A standard transversality argument allows us to extend $\tilde{s}$ to a section $\tilde{S}$ of $\nu(\tilde{\iota})$ which is transverse to the 0 -section and which satisfies the following two conditions:

- If $x$ is a double point of $\tilde{\iota}$ then $\tilde{S}(x) \neq 0$.

- If $\tilde{S}(x)=0$ then $\tilde{S}(T(x)) \neq 0$.

Let $\Delta \subset \mathbb{R}^{3 n}$ denote the closure of the double points of $f$. Then $\Delta$ is an immersed submanifold with boundary $\Sigma$ and $\Delta$ has triple points at the triple points of $f$. Let $\Delta_{\text {res }}$ denote the resolution of $\Delta$ and let $\iota: \Delta_{\text {res }} \rightarrow \mathbb{R}^{3 n}$ denote the natural immersion with image $\Delta$. Let $\nu(\iota)$ denote the normal bundle of the immersion $\iota$. Note that there is a natural map $\Pi: \tilde{\Delta}_{\text {res }} \rightarrow \Delta_{\text {res }}$ which is a double cover of $\Delta_{\text {res }}-\Sigma$ when restricted to $\tilde{\Delta}_{\text {res }}-\tilde{\Sigma}$, and which maps $\tilde{\Sigma}$ diffeomorphically onto $\Sigma$.

Define the section $S$ of $\nu(\iota)$ as follows:

$$
\begin{aligned}
& S(y)= \\
& \begin{cases}d f\left(\tilde{S}\left(y_{1}\right)\right)+d f\left(\tilde{S}\left(y_{2}\right)\right) & \text { if } y \in \Delta_{\text {res }}-\Sigma, \text { where } y_{1} \neq y_{2}, \Pi\left(y_{1}\right)=\Pi\left(y_{2}\right)=y, \\
2 d f\left(\tilde{S}\left(y_{1}\right)\right) & \text { if } y \in \Sigma, \text { where } \Pi\left(y_{1}\right)=y .\end{cases}
\end{aligned}
$$

Let $C(\Sigma) \subset \Delta_{\text {res }}$ be a small open collar on the boundary $\Sigma$ of $\Delta_{\text {res. }}$. Let $\Delta^{\prime \prime}$ denote the image of the immersion $y \mapsto \iota(y)+\epsilon S(y), y \in \Delta_{\text {res }}-C(\Sigma)$ for some small $\epsilon>0$. Then, if $\epsilon$ and the collar $C(\Sigma)$ are small enough, $\Delta^{\prime \prime}$ is a chain with boundary $\partial \Delta^{\prime \prime}=\Sigma^{\prime \prime}$ satisfying $\Sigma^{\prime \prime} \cap f(M)=\emptyset$. If $l_{2}$ denotes the mod 2linking number, • denotes the mod 2-intersection number, and $\sharp(F)$ denotes the mod 2-number of elements in the finite set $F$, then

$$
\operatorname{lk}_{2}\left(\Sigma^{\prime \prime}, f(M)\right)=\Delta^{\prime \prime} \bullet f(M)=\sharp\left(\tilde{S}^{-1}(0)\right)+t_{2}(f),
$$




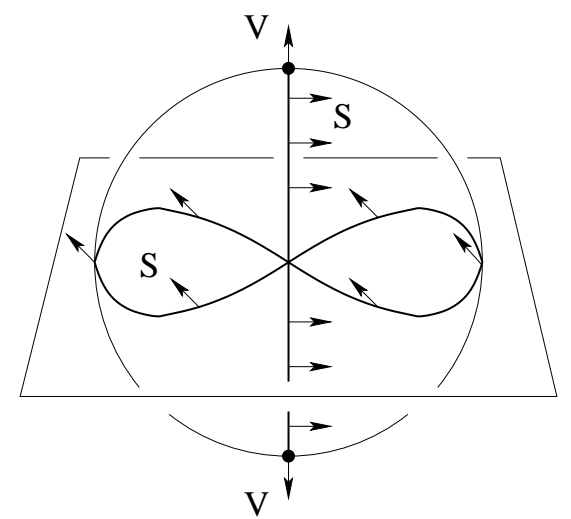

Figure 1. A piece of $f(M)$ (represented by a 2-sphere and a piece of a plane) with the double point set $\Delta$ (fat lines), its normal field $S$, and singularity set $\Sigma$ (dots) with its outward normal field $V$ in $\Delta$.

since near each zero $z$ of $\tilde{S}$ there is a unique intersection point of $\Delta^{\prime \prime}$ and $f(M)$ near $f(z)$, and near each triple point of $f$ there are exactly three such intersection points.

The homology class of the cycle $\tilde{\Delta}$ in $M$ is Poincaré dual to $n^{\text {th }}$ normal StiefelWhitney class $\bar{w}_{n}$ of $M$, see [6]. Thus

$$
\bar{w}_{n}^{2}[M]=\tilde{\Delta} \bullet \tilde{\Delta}=\sharp\left(\tilde{S}^{-1}(0)\right),
$$

since the image of a slight shift of the immersion $\tilde{\iota}$ along $\tilde{S}$ intersects $\tilde{\Delta}$ near each zero of $\tilde{S}$ and in two points near each double point of $\tilde{\iota}$.

Equations (4) and (5) imply

$$
\mathrm{lk}_{2}\left(\Sigma^{\prime \prime}, f(M)\right)=\bar{w}_{n}^{2}[M]+t_{2}(f) .
$$

Recall that $\Sigma^{\prime} \subset \mathbb{R}^{3 n}$ is the submanifold which results when $\Sigma$ is shifted slightly along its unit outward normal vector field $V$ in $\Delta$, and that $\Sigma^{\prime} \cap f(M)=\emptyset$. We compare the linking numbers $\mathrm{lk}_{2}\left(\Sigma^{\prime \prime}, f(M)\right)$ and $\mathrm{lk}_{2}\left(\Sigma^{\prime}, f(M)\right)$ :

Let $\tilde{\Sigma}_{0} \subset M$ be the submanifold which results when $\tilde{\Sigma}$ is shifted a small distance along $\tilde{S}$. Let $\Sigma_{0}=f\left(\tilde{\Sigma}_{0}\right)$ and for $p \in \Sigma$, let $p_{0}=f\left(\tilde{p}_{0}\right)$ where $\tilde{p}_{0}$ is the point in $\tilde{\Sigma}_{0}$ corresponding to $\tilde{p} \in \tilde{\Sigma}$ with $f(\tilde{p})=p$.

For small $\epsilon>0$ and $p \in \Sigma$ let $l_{p}(\epsilon)$ be the segment of the straight line through $p+\epsilon V(p)$ and $p_{0}$ of length $2 \epsilon$ and centered at $p_{0}$. For $\epsilon>0$ and the shifting of $\tilde{\Sigma}$ in $M$ small enough,

$$
\Gamma=\bigcup_{p \in \Sigma} l_{p}(\epsilon)
$$

is a submanifold of $\mathbb{R}^{3 n}$. If the collar $C(\Sigma)$ is chosen small enough and if the 
shifting distance along $S$ is small enough then the boundary $\partial \Gamma$ of $\Gamma$ is isotopic to $\Sigma^{\prime} \cup \Sigma^{\prime \prime}$ in $\mathbb{R}^{3 n}-f(M)$. Thus

$$
\mathrm{lk}_{2}\left(\Sigma^{\prime}, f(M)\right)=\mathrm{lk}_{2}\left(\Sigma_{0}, f(M)\right)+\Gamma \bullet f(M)=\mathrm{lk}_{2}\left(\Sigma^{\prime \prime}, f(M)\right)+\Gamma \bullet f(M) .
$$

We compute $\Gamma \bullet f(M)$ : The intersection $\Gamma \cap f(M)$ is a clean intersection. That is, $\Gamma \cap f(M)=\Sigma_{0}$ is a manifold and the tangent bundle

$$
T \Sigma_{0}=T f(M) \cap T \Gamma \subset T \mathbb{R}^{3 n},
$$

where all bundles in the left hand side are restricted to $\Sigma_{0}$.

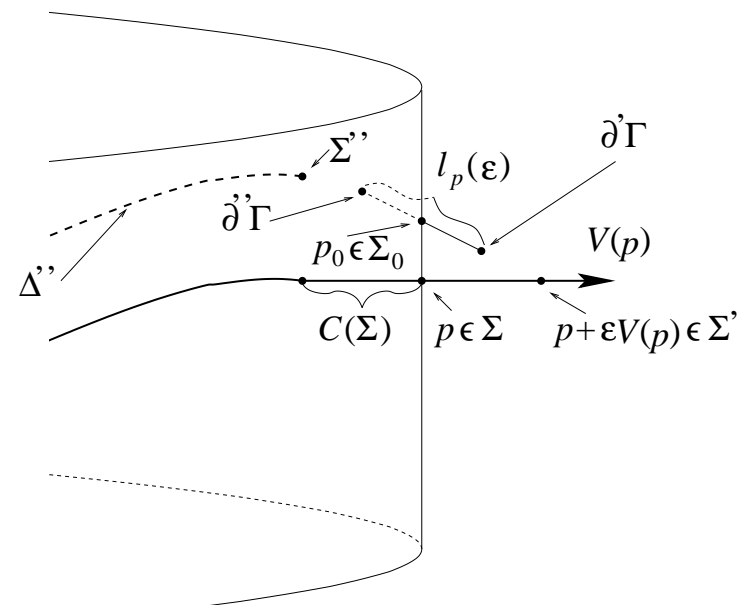

Figure 2. The normal space of $\Sigma$ in $\mathbb{R}^{3 n}$ at $p \in \Sigma$. In the figure the boundary of $\Gamma$ is the union of $\partial^{\prime} \Gamma$, isotopic to $\Sigma^{\prime}$ in $\mathbb{R}^{3 n}-f(M)$, and $\partial^{\prime \prime} \Gamma$ isotopic to $\Sigma^{\prime \prime}$ in $\mathbb{R}^{3 n}-f(M)$.

As in [4], we find

$$
\Gamma \bullet f(M)=w_{n-1}(\xi)
$$

where $\xi$ is the so called excess bundle over $\Sigma_{0}$ :

$$
\xi=T \mathbb{R}^{3 n} /(T \Gamma+T f(M)),
$$

where all bundles are restricted to $\Sigma_{0}$.

To finish the proof it remains to calculate $w_{n-1}(\xi)$. Note that

$$
T \Gamma \mid \Sigma_{0}=T \Sigma_{0} \oplus \epsilon^{1},
$$

where $\epsilon^{1}$ is the trivial line bundle directed along the intervals $l_{p}(\epsilon)$. Thus, by (8),

$$
\xi \oplus T f(M)\left|\Sigma_{0} \oplus \epsilon^{1}=T \mathbb{R}^{3 n}\right| \Sigma_{0} .
$$

The bundle $T f(M) \mid \Sigma_{0}$ is identified with $T M \mid \tilde{\Sigma}_{0}$ by the differential of $f$. Hence if $i_{0}: \tilde{\Sigma}_{0} \rightarrow M$ denotes the inclusion then $w(\xi)=i_{0}^{*} \bar{w}(M)$. Therefore, if $F_{V}$ denotes 
the fundamental homology class of the manifold $V$ and PD denotes the Poincaré duality operator,

$$
\begin{aligned}
\left\langle w_{n-1}(\xi), F_{\Sigma_{0}}\right\rangle & =\left\langle i_{0}^{*} \bar{w}(M), F_{\tilde{\Sigma}_{0}}\right\rangle=\left\langle\bar{w}(M), i_{0 *}\left(F_{\tilde{\Sigma}_{0}}\right)\right\rangle=\left\langle\bar{w}(M), \operatorname{PD} \bar{w}_{n+1}(M)\right\rangle \\
& =\left\langle\bar{w}(M) \cup \bar{w}_{n+1}(M), F_{M}\right\rangle=\bar{w}_{n-1} \bar{w}_{n+1}[M]
\end{aligned}
$$

Here, the third equality follows from the well-known formula $\mathrm{PD} \bar{w}_{n+1}(M)=i_{*} F_{\tilde{\Sigma}}$, where $i: \tilde{\Sigma} \rightarrow M$ denotes the inclusion, together with $i_{*} F_{\tilde{\Sigma}}=i_{0 *} F_{\tilde{\Sigma}_{0}}$. Equations (6), (7), and (10) prove the theorem.

\section{Proof of Theorem 3}

Let $\mathfrak{N}_{*}, \Omega_{*}$, and $\Omega_{*}^{U}$ denote the cobordism ring, the oriented cobordism ring, and the complex cobordism ring, respectively. Note that there are natural forgetting homomorphisms

$$
\Omega_{*}^{U} \longrightarrow \Omega_{*} \longrightarrow \mathfrak{N}_{*} .
$$

For a manifold $M$, let $[M]$ denote its cobordism class.

Using some facts from cobordism theory which can all be found in Chapter 4 of Stong's book [8], we show that it is enough to prove the theorem for oriented $4 k$-manifolds $M$ such that either

(a) $[M] \in \Omega_{4 k}$ maps to a square $[N \times N] \in \mathfrak{N}_{4 k}$, or

(b) $[M]$ is a torsion element of $\Omega_{4 k}$ (in fact, $[M]$ torsion implies $2 \cdot[M]=0$ ):

Let $\operatorname{Tors}\left(\Omega_{*}\right)$ denote the torsion subgroup of $\Omega_{*}$. The homomorphism $\Omega_{*}^{U} \rightarrow \Omega_{*}$ induces an epimorphism

$$
\Omega_{*}^{U} \longrightarrow \Omega_{*} / \operatorname{Tors}\left(\Omega_{*}\right) .
$$

and the image $\Omega_{*}^{U} \rightarrow \mathfrak{N}_{*}$ consists of squares of elements in $\mathfrak{N}_{*}$.

Hence, if $M$ is any oriented $4 k$-manifold then there exists some oriented $4 k$ manifold $V$ such that $[V]$ is torsion in $\Omega_{4 k}$ and $[M]+[V]=[N \times N]$ in $\mathfrak{N}_{4 k}$. This implies that the theorem follows once it is proved for manifolds satisfying (a) or (b) above.

First consider (a): let $M=N \times N$. Then $\bar{w}(M)=\bar{w}(N) \times \bar{w}(N)$ and hence

$$
\bar{w}_{2 k+1}(M)=\sum_{i+j=2 k+1} \bar{w}_{i}(N) \times \bar{w}_{j}(N) .
$$

Thus

$$
\begin{aligned}
& \left\langle\bar{w}_{2 k_{1}+1}(M) \ldots \bar{w}_{2 k_{r}+1}(M), F_{M}\right\rangle= \\
& =\sum\left\langle\bar{w}_{i_{1}}(N) \ldots \bar{w}_{i_{r}}(N), F_{N}\right\rangle \cdot\left\langle\bar{w}_{j_{1}}(N) \ldots \bar{w}_{j_{r}}(N), F_{N}\right\rangle .
\end{aligned}
$$

Since $i_{s}+j_{s}$ is odd for all $i_{s}, j_{s}$ there is a fixed point free involution $T$ acting on 
the set of the terms in the sum in (11) such that $i_{1}+\cdots+i_{r}=2 k=j_{1}+\cdots+j_{r}$ :

$$
\begin{aligned}
T: & \left\langle\bar{w}_{i_{1}}(N) \ldots \bar{w}_{i_{r}}(N), F_{N}\right\rangle \cdot\left\langle\bar{w}_{j_{1}}(N) \ldots \bar{w}_{j_{r}}(N), F_{N}\right\rangle \\
& \mapsto\left\langle\bar{w}_{j_{1}}(N) \ldots \bar{w}_{j_{r}}(N), F_{N}\right\rangle \cdot\left\langle\bar{w}_{i_{1}}(N) \ldots \bar{w}_{i_{r}}(N), F_{N}\right\rangle .
\end{aligned}
$$

Thus the terms in the left hand side of (11) which does not vanish for dimensional reasons cancel in pairs and hence $\bar{w}_{2 k_{1}+1} \ldots \bar{w}_{2 k_{r}+1}[M]=0$.

Next consider (b): let $u: \mathfrak{N}_{4 k} \rightarrow \mathbb{Z}_{2}$ denote the homomorphism induced by the product of odd-dimensional normal Stiefel-Whitney classes $\bar{w}_{2 k_{1}+1} \ldots \bar{w}_{2 k_{r}+1}$, $\sum 2 k_{j}+1=4 k$. Odd-dimensional Stiefel-Whitney classes are mod 2-reductions of twisted integer classes, see [3], p. 140. Hence, a product of an even number of such classes is an integer class so the map

$$
\Omega_{4 k} \stackrel{\pi}{\longrightarrow} \mathfrak{N}_{4 k} \stackrel{u}{\longrightarrow} \mathbb{Z}_{2}
$$

lifts to a homomorphism

$$
\Omega_{4 k} \stackrel{U}{\longrightarrow} \mathbb{Z}
$$

Thus $U$ and therefore $u \circ \pi$ is zero on any torsion element of $\Omega_{4 k}$.

\section{References}

[1] T. Ekholm and A. Szücs, Geometric formulas for Smale invariants of codimension two immersions, Topology, to appear.

[2] T. Ekholm and A. Szücs, The group of immersions of homotopy $4 k-1$-spheres, preprint.

[3] J. Milnor and J. Stasheff, Characteristic classes, Ann. of Math. Stud. 76, Princeton Univ. Press, 1974.

[4] D. Quillen, Elementary proof of some results of cobordism theory using Steenrod operations, Adv. in Math. 7 (1971), 29-56.

[5] F. Ronga, On multiple points of smooth immersions Comment. Math. Helv. 55 (1980), $521-527$.

[6] F. Ronga, La classe duale aux points doubles d'une application Composito Math. 27 (1973), $223-232$.

[7] O. Saeki, A. Szűcs and M. Takase, Regular homotopy classes of immersions of 3-manifolds into 5-space, Manuscripta Math., to appear.

[8] R. Stong, Notes on cobordism theory, Princeton Univ. Press, 1968.

9] A. Szücs, The linking number of singular maps, Comment. Math. Helv. 61 (1986), 360-369.

[10] H. Whitney, The self-intersections of a smooth $n$-manifold in $2 n$-space, Ann. of Math. 45 (1944), 220-293.

Tobias Ekholm

Uppsala University

Department of Mathematics

P.O. Box 480

SE-751 06 Uppsala

Sweden

e-mail: tobias@math.uu.se

(Received: October 12, 2001)

\author{
A. Szücs \\ Eötvös Loránd University \\ Department of Analysis \\ Pázmány Péter sétány 1/C \\ H-1117 Budapest \\ Hungary \\ e-mail: szucs@cs.elte.hu
}

